\title{
Development of Picosecond 755-nm Alexandrite Laser for Treatment of Skin Aging
}

\author{
Seokwon Oh, MS \\ Hanyoung Ryu, PhD \\ Youngseok Seo, PhD
}

R\&D Center, Wontech Co., Ltd., Daejeon, Korea

Received March 13, 2020

Revised March 30, 2020

Accepted April 10, 2020

\section{Correspondence}

Youngseok Seo

R\&D Center, Wontech Co., Ltd., Daejeon 34028, Korea

E-mail: Physys@wtlaser.com

https://orcid.org/0000-0001-7939-5428

(C) Korean Society for Laser, Dermatology and Trichology

(a) This is an open access article distributed under the terms of the Creative Commons Attribution NonCommercial License (http://creativecommons.org/ licenses/by-nc/4.0) which permits unrestricted noncommercial use, distribution, and reproduction in any medium, provided the original work is properly cited.
We have developed a picosecond $755 \mathrm{~nm}$ alexandrite laser system. To generate picosecond laser pulses, we used self-injection techniques including Q-switch and cavity dump without mode-locking method. We have achieved through a comprehensive combination of complex optical technique, thermodynamic design, high power design, high-speed driver, and precise control technique. The uniform energy of the beam profile allows a safe and effective treatment outcome to be predicted.

Key words

Picosecond laser, Alexandrite laser, Picowon, Removing tattoos, Pigmented lesions

Supplementary video fille: This article contains supplementary material (It is available at https://youtu.be/71 mHVOnxHwM). 


\section{INTRODUCTION}

In the past decades, lasers with shorter pulses have been developed in a variety of industries including medical, defense, semiconductor, and manufacturing. Since the report of selective photothermolysis, various medical laser devices have been developed to more precisely achieve the challenge of treating pigmented lesion [1]. In dermatology, picosecond lasers treat lesions with $1 / 3$ to $1 / 2$ of the energy used in nanosecond lasers. Picosecond lasers have more photoacoustic effects and less photothermal effects, compared to nanosecond lasers [2,3]. Therefore, the risk of collateral damages to surrounding tissue is less. As more people want to obtain younger and healthier appearing face themselves, picosecond laser treatment is a quickly growing field in cosmetic dermatology.

A $755 \mathrm{~nm}$ alexandrite picosecond laser device, Cynosure's Picosure $^{\mathrm{TM}}$, was first approved by U.S. food and drug administration (FDA) in December 2012 [4]. Alexandrite picosecond laser is used for skin rejuvenation, removing tattoos, treating a scar, and pigmented lesions [5,6]. The laser gain medium of alexandrite $\left(\mathrm{Cr}^{3+}: \mathrm{BeAl}_{2} \mathrm{O}_{4}\right)$ lasers is an alexandrite crystal doped with chromium ions ( $\mathrm{Cr} 3+)$. One has to consider that in contrast to Nd:YAG lasers, alexan-

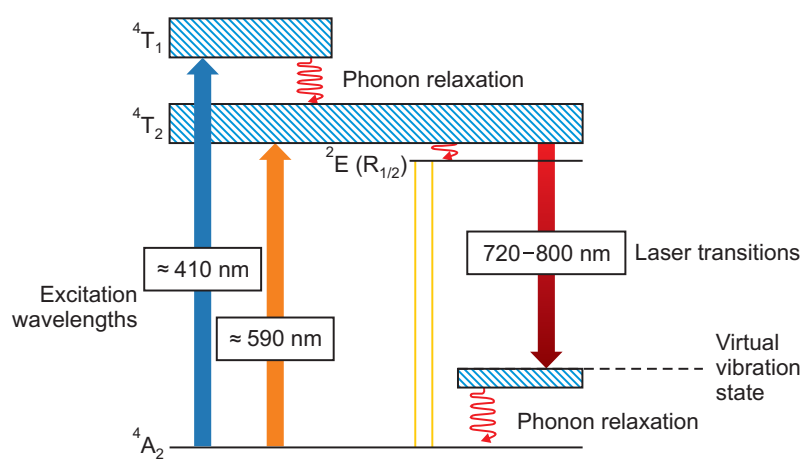

Fig. 1. Energy levels and laser transition of the quasi-four-level alexandrite laser [7]. drite lasers are not four-level lasers but are operated in a quasi-four-level regime, as shown in Fig. 1 [7]. Alexandrite laser can be operated at wavelengths in the range from 700 to $800 \mathrm{~nm}$, in many cases around $755 \mathrm{~nm}$, where the maximum laser gain can be achieved. Alexandrite also has a large gain variation depending on temperature, and polarization-dependent absorption [8,9].

To generate a picosecond laser pulse, we used the selfinjection technique, including Q-switch and cavity dump, without mode-locking method [10]. A high voltage and ultra-fast driver are required for cavity dumping. Laser head was composed of alexandrite crystal rod, Pockels cell, polarizer, high reflective coating mirror, and an output coupler (partially-reflective mirror), as shown in Fig. 2. Dual Xenon flash lamps were used as a light pumping source. To maintain the uniform temperature of alexandrite crystal and flash lamp, the water-cooling system and heater were designed and applied.

\section{RESULTS AND CONCLUSION}

We have been developed a picosecond $755 \mathrm{~nm}$ Alexandrite laser system, and named the system "PICOWON". The laser output pulse width can be adjusted by varying amplitude (voltage) and width (picosecond) in the pulse generator. The developed laser typically can be operated at pulse width in the range from 600 to 800 ps. Fig. 3 shows the laser output pulse width measured using a digital oscilloscope (DPO 4104B/1GHz 5GS/s, Tektronix) and silicon pho-

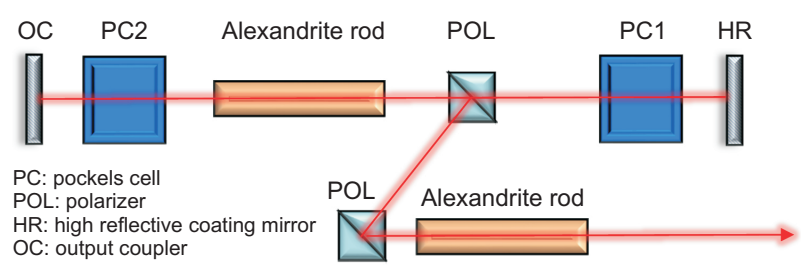

Fig. 2. Schematic diagram of the laser cavity.
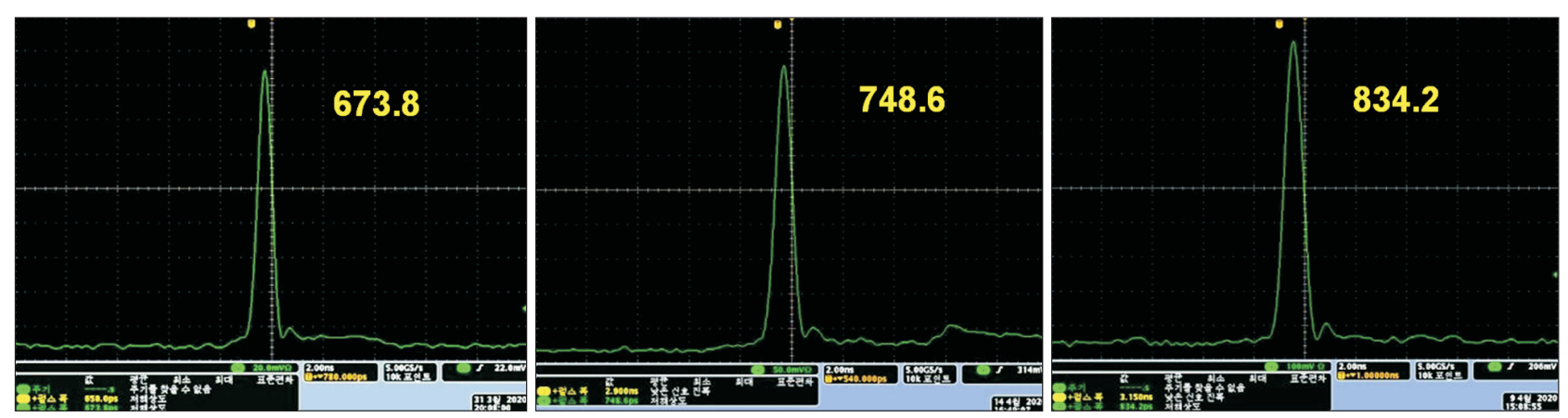

Fig. 3. Measured laser output pulse width. 
 \\ AESTHETICS}
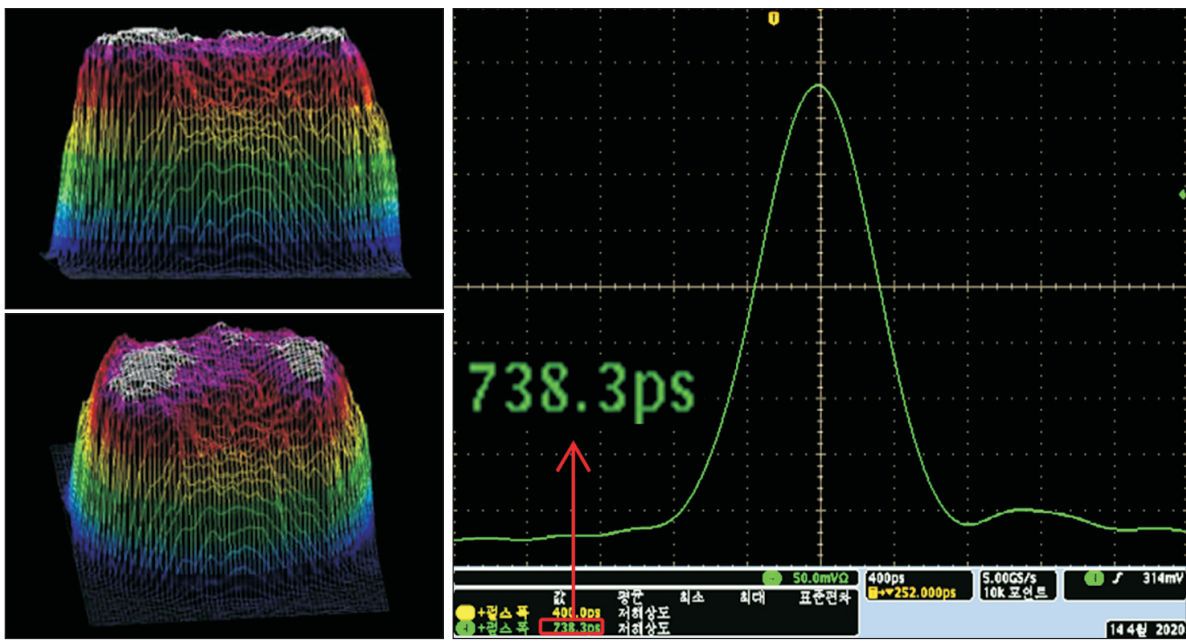

Fig. 4. Measured laser output beam profile.

Table 1. Developed laser system specifications

\begin{tabular}{ll}
\hline Specifications & \multicolumn{1}{c}{ Value } \\
\hline Wavelength & $755 \mathrm{~nm}$ \\
Laser type & Picosecond Alexandrite Laser \\
Pulse energy & $230 \mathrm{~mJ}$ \\
Pulse width & $600-800 \mathrm{ps}$ \\
Peak power & $0.33 \mathrm{G} \mathrm{W}(700 \mathrm{ps})$ \\
Pulse rate & $1-10 \mathrm{~Hz}$ \\
Spot size & $2,3,3.5,4,5,5.5,6,8 \mathrm{~mm}$ \\
Start up time & $<15 \mathrm{minutes}$ \\
Dimensions & $500(\mathrm{~W}) * 960(\mathrm{D}) * 900(\mathrm{H})$ \\
Weight & $100 \mathrm{Kg}$ \\
\hline
\end{tabular}

todetector (ET-2030, EOT). The maximum average output energy of $230 \mathrm{~mJ}$ was achieved in the picosecond regime. To increase the repetition rate, the thermal lens effect must be compensated. Therefore, we performed a task to stabilize the temperature of the laser head and increased the repetition rate to $10 \mathrm{~Hz}$. Fig. 4 shows the beam profile measured using laser cam-HR (1098577, Coherent). It shows a top hat-shaped beam pattern and means a uniform spatial distribution.

Table 1 shows features of developed $755 \mathrm{~nm}$ picosecond laser specifications. We developed two types of handpieces, which are zoom and MLA (Micro Lens Array) handpiece with spot size in the range from 2 to $8 \mathrm{~mm}$.

\section{DISCUSSION}

Wontech developed the first commercially available alexandrite picosecond laser in Korea, as shown in Fig. 5. To make a high-efficiency alexandrite picosecond laser, it can be achieved through a comprehensive combination of com-

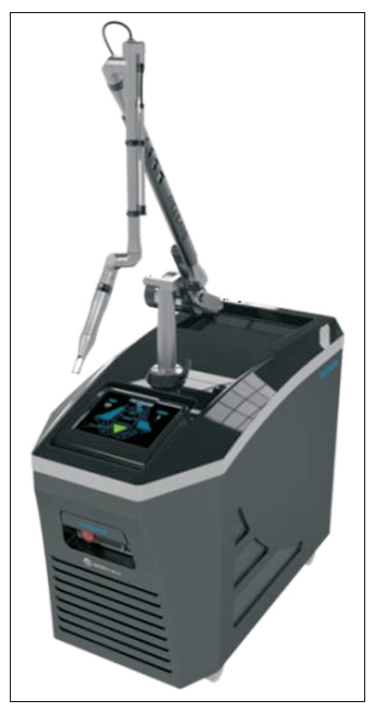

Fig. 5. Developed picosecond laser (PICOWON).

plex optical technique, thermodynamic design, high power design, high-speed driver, and precise control technique. High peak power in shorter pulse duration provides better efficacy in treatments of pigments, tattoo removal. Uniform energy of the beam profile allows safe and effective treatment results to be predicted.

\section{CONFLICT OF INTEREST}

No potential conflict of interest relevant to this article was reported.

\section{REFERENCES}

1. Anderson RR, Parrish JA. Selective photothermolysis: precise microsurgery by selective absorption of pulsed radiation. Sci- 
ence 1983;220:524-7.

2. Saki N. Picosecond laser applications in aesthetic dermatology. J Surg Dermatol 2017;2(T1):107-8.

3. Ge Y, Yang Y, Guo L, Zhang M, Wu Q, Zeng R, et al. Comparison of a picosecond alexandrite laser versus a $Q$-switched alexandrite laser for the treatment of nevus of Ota: a randomized, split-lesion, controlled trial. J Am Acad Dermatol 2020;83:397-403.

4. Cynosure. Cynosure receives FDA clearance for PicoSure ${ }^{\mathrm{TM}}$, the first picosecond device to remove tattoos and benign pigmented lesions [online]. [cited 2020 Jun 22]; Available from: https://www.prnewswire.com/news-releases/ cynosure-receives-fda-clearance-for-picosure-the-first-picosecond-device-to-remove-tattoos-and-benign-pigmentedlesions-181993511.html

5. Zhang $\mathrm{M}$, Fang J, Wu Q, Lin T. Evaluation of the safety and efficacy of a picosecond alexandrite laser with DLA for acne scars in Chinese patients. Lasers Surg Med 2020;52:176-81.

6. Alegre-Sanchez $A$, Jiménez-Gómez $N$, Moreno-Arrones ÓM, Fonda-Pascual P, Pérez-García B, Jaén-Olasolo P, et al. Treatment of flat and elevated pigmented disorders with a 755$\mathrm{nm}$ alexandrite picosecond laser: clinical and histological evaluation. Lasers Med Sci 2018;33:1827-31.

7. Wieneke S, Gerhard C. Lasers in Medical Diagnosis and Therapy: Basics, Applications and Future Prospects. Bristol: IOP Publishing; 2018.

8. Guch S Jr, Jones CE. Alexandrite-laser performance at high temperature. Opt Lett 1982;7:608-10.

9. Vinnik DA, Zherebtsov DA, Archugov SA, Bischoff M, Niewa R. Crystal growth and characterization of alexandrite. Cryst Growth Des 2012;12:3954-6.

10. Brito Cruz CH, Palange E, De Martini F. A study of the selfinjected laser for subnanosecond pulse generation. Appl Phys B 1984;35:95-104. 